\title{
Icaritin inhibits decidualization of endometrial stromal cells
}

\author{
AIWEN LE ${ }^{1}$, ZHONG HAI WANG $^{1}$, XIAO YUN DAI $^{1}$, TIAN HUI XIAO ${ }^{1}$, \\ RONG ZHUO $^{1}$, BAOZHEN ZHANG ${ }^{2}$, ZHONGLIN XIAO $^{2}$ and XIUJUN FAN ${ }^{2}$

\begin{abstract}
${ }^{1}$ Department of Obstetrics and Gynecology, Affiliated Shenzhen Nanshan People's Hospital of Guangdong Medical University, Shenzhen, Guangdong 518052; ${ }^{2}$ Laboratory for Reproductive Health, Shenzhen Institutes of Advanced Technology,
\end{abstract} \\ Chinese Academy of Sciences, Shenzhen, Guangdong 518055, P.R. China
}

Received November 28, 2016; Accepted July 28, 2017

DOI: $10.3892 / \mathrm{etm} .2017 .5278$

\begin{abstract}
The aim of the present study was to investigate the effects of Icaritin on the proliferation and decidualization of endometrial stromal cells (ESCs). A total of 20 specimens of endometrium were collected during hysterectomy at the Gynecology Department of Shenzhen Nanshan People's Hospital (Shenzhen, China) between August 2014 and December 2015. The endometrium was digested with high concentrations of collagenase and DNase and filtered with meshes, and then the glandular epithelial and stromal cells were separated by the adhesion purification method. The purity of stromal cells was identified by vimetin and cytokeratin 7 immunostaining. The estradiol + progesterone $(\mathrm{E} 2+\mathrm{P} 4)$ and/or cyclic adenosine monophosphate (cAMP) were added to induce an in vitro decidualization model, which was used to analyze the effect of Icaritin on the decidualization ability of the human ESCs. The decidualization markers of human ESCs, prolactin (PRL) and insulin-like growth factor-binding protein 1 (IGFBP-1), was analyzed by reverse-transcription quantitative polymerase chain reaction measurements of the mRNA levels, PRL immunostaining and ELISA analysis of the IGFBP-1 protein levels in the cells or cell culture supernatant separately. The results demonstrated that treatment with E2+P4 and/or cAMP for $96 \mathrm{~h}$ was able to induce decidualization in ESCs, and that the cells demonstrated polygon-shaped epithelioid changes. The cell nuclei revealed multinuclear changes, and the cells were also observed to be large and round in shape. The PRL expression and upregulated IGFBP-1 mRNA and
\end{abstract}

Correspondence to: Dr Aiwen Le, Department of Obstetrics and Gynecology, Affiliated Shenzhen Nanshan People's Hospital of Guangdong Medical University, 89 Taoyuan Road, Shenzhen, Guangdong 518052, P.R. China

E-mail: leaiwen@126.com

Dr Xiujun Fan, Laboratory for Reproductive Health, Shenzhen Institutes of Advanced Technology, Chinese Academy of Sciences, 1068 Xueyuan Boulevard, Shenzhen, Guangdong 518055, P.R. China E-mail:xj.fan@siat.ac.cn

Key words: endometrial stromal cells, decidualization, Icaritin protein levels in the $\mathrm{E} 2+\mathrm{P} 4+\mathrm{cAMP}$ treatment group indicated successful decidualization of the in vitro model. However, the addition of Icaritin inhibited the expression of PRL and IGFBP-1 mRNA, as well as IGFBP-1 protein in the induced ESCs compared with groups without Icaritin. These results suggest that Icaritin was able to inhibit the expression of decidualization-related genes in ESCs in vitro. However, the exact mechanisms require further investigation.

\section{Introduction}

Endometrium with a thickness $<8 \mathrm{~mm}$ in the middle luteal phase (6-10 days following ovulation) is considered as thin endometrium (1), which is a potential cause of infertility. Hysteroscopy has been reported to demonstrate no adhesion in the pelvic cavity as the endometrium is smooth and thin in patients with thin endometrium, which may affect the implantation of blastocysts in the endometrium $(2,3)$. There are a number of potential factors that may lead to thin endometrium, with decidualization failure being one of the major causes $(4,5)$.

Under the effects of estrogen and progestogen, endometrial stromal cells (ESCs) are able to undergo a typical transformation, including morphological, biochemical and vascular system changes, which are termed as endometrial decidualization (6). Endometrial decidualization is one of the essential preconditions for blastocyst implantation, placentation and pregnancy maintenance (5). Furthermore, the morphology of human ESCs in decidualization is altered from spindle to polygon shape, the cell becomes larger and round, the nucleus becomes large and pale, the cytoplasm is rich in glycogen and lipid droplets and the cells reveal epithelioid changes (7). In addition, the intercellular boundaries become unclear as prolactin (PRL), insulin-like growth factor-binding protein 1 (IGFBP-1), tissue factors, neuropeptide, and extracellular matrix components are secreted from the cells, among which PRL and IGFBP-1 are considered as the markers of ESC decidualization (8-10). Previous studies have revealed that PRL is expressed in gestational endometrium and decidual cells induced in vitro (10), and that IGFBP-1 is overexpressed in decidualized endometrium stroma and positively associated with the degree of stromal cell decidualization (11). Additionally, the expression of IGFBP-1 in the decidualized endometrium is regulated by the progesterone receptor (PR) (12). 
Phytoestrogen is a plant-derived non-steroidal compound (13). The structure and biological activity of phytoestrogen are similar to those of endogenous estrogen (13). However, phytoestrogen has bidirectional regulatory effects: It demonstrates anti-estrogenic activity when the level of estrogen is high in the body, and reveals estrogenic activity when the level of estrogen is relatively low (14). Icaritin is one of the major active ingredients of epimedium. Icaritin and its derivative, desmethylicaritin, exhibit evident estrogenic effects, and are able to increase the expression of PR (15). However, only a small number of studies to date have investigated the effects of Icaritin on endometrial cells and its role in decidualization of the cells. Therefore, the effect of Icaritin on primary ESCs was investigated in the present study.

\section{Materials and methods}

Human endometrium. In total, 20 endometrial tissues were obtained during hysterectomy at the Shenzhen Nanshan People's Hospital (Shenzhen, China) between August 2014 and December 2015, with written informed consent obtained from all patients (aged 30-39), following the approved procedures of the Institutional Review Board of Shenzhen Nanshan People's Hospital. Subsequently, the collected endometrium samples were used for the separation and culture of primary ESCs. The T HESC human ESC cell line was provided by Professor Haibin Wang of the Institute of Zoology, Chinese Academy of Sciences (Beijing, China) and used for the induction of the decidualization model. The study was approved by the Ethics Committee of Shenzhen Institutes of Advanced Technology, Chinese Academy of Sciences (Shenzhen, China).

Isolation, culture and identification of primary ESCs ( $p E S C s$ ). Fresh endometrial tissues were obtained during hysterectomy, placed in ice-cold sterilized PBS and transferred to the laboratory for the separation and culture of pESCs within $1 \mathrm{~h}$. Tissues were minced and digested with high concentrations of collagenase $(1 \mathrm{mg} / \mathrm{ml})$ and DNase $(0.2 \mu \mathrm{g} / \mathrm{ml})$ for $1 \mathrm{~h}$ at $37^{\circ} \mathrm{C}$, and then filtered through a mesh. The glandular epithelial and stromal cells were separated by the adhesion purification method, as described previously (16).

The stromal cells reached confluence after 3 days of culture in the complete culture medium (cDMEM, high glucose Dulbecco's modified Eagle's medium with 10\% fetal bovine serum; Invitrogen; Thermo Fisher Scientific, Inc., Waltham, $\mathrm{MA}, \mathrm{USA}$ ) at $37^{\circ} \mathrm{C}$ with $5 \% \mathrm{CO}_{2}$. Next, the culture medium was discarded, cells were washed with PBS, and $0.25 \%$ trypsin and $0.53 \mathrm{mM}$ EDTA solution (Invitrogen; Thermo Fisher Scientific, Inc.) was added to disassociate the cells for $2 \mathrm{~min}$ at room temperature, and the cells were gently resuspended. The cell suspension was transferred to a centrifuge tube and centrifuged at $230 \mathrm{x}$ g for $5 \mathrm{~min}$ at room temperature. The supernatant was then discarded and the fresh culture medium was added to resuspend the cell pellet gently. The cells were subsequently split at a ratio of 1:3.

For the purity identification of the primary ESCs, they were passaged three times, and were then seeded into a 24 -well culture plate with a density of $1 \times 10^{4}$ cells/ml. Once the cells had reached $80 \%$ confluence, the culture medium was discarded, and the cells were washed with PBS twice. Next, $1 \mathrm{ml}$ ice-cold $4 \%$ paraformaldehyde (PFA) was added into each well to fix the cells for $20 \mathrm{~min}$ on ice. Finally, the fixed cells were washed with PBS and stored at $4^{\circ} \mathrm{C}$ in PBS for immunoflurescence identification with vimentin and cytokeratin 7 (CK7) as described below.

In vitro decidualization model of human ESCs. Induction of the decidualization of the stromal cells $\left(10^{5}\right.$ cells $)$ was performed following with estradiol $(\mathrm{E} 2,10 \mathrm{nM})+$ progesterone (P4; $1 \mu \mathrm{M})$ (both from Sigma-Aldrich; Merck KGaA, Darmstadt, Germany) and/or cAMP $(0.5 \mathrm{mM})$ using an induction method, as previously reported (14). Briefly, the confluence ESCs from the third passage after thaw were used to induce decidualization with different treatment groups: PE (P4+E2), PEC (P4+E2+cAMP), PC (P4+cAMP) and control (complete culture medium) groups. The concentrations of E2, P4 and cAMP were selected according to a previous study (17).

Following the successful induction of the decidualization model, Icaritin (10 $\mu \mathrm{M}$; Beijing Shenogen Pharma Group, Beijing, China) was added to the induction cocktail to investigate its effect with the following groups: PI (P4+Icaritin), PE (P4+E2), PEI (P4+E2+Icaritin), PEC (P4+E2+cAMP), PECI (P4+E2+cAMP+Icaritin), PC (P4+cAMP), PCI (P4+cAMP+Icaritin) and the control (complete culture medium) groups. Then, the induced decidualization was performed for all groups as described above.

Each group contained triplicates, and each condition had been performed three times. Cells from different treatment groups were all observed and photographed with an inverted microscope during the treatment period. In all treatments, following $96 \mathrm{~h}$ of induction, the culture media from different treatment groups were collected and stored at $-80^{\circ} \mathrm{C}$ for IGFBP-1 ELISA analysis later. Furthermore, a number of cells from different groups were harvested for total RNA extraction, and a number of the cells were fixed with 4\% PFA at room temperature for $15 \mathrm{~min}$, then washed with PBS and stored at $4^{\circ} \mathrm{C}$ in PBS for immunofluorescence staining.

Reverse-transcription-quantitative polymerase chain reaction $(R T-q P C R)$. The expression of PRL and IGFBP-1 mRNA in decidualized ESC cells was measured by RT-qPCR according to the previously described methods (18). Primer sequences were as follows: PRL sense, ATGGAAGTCCCGACCAGAC and antisense, ATTGAGGAGCAAACCAAACG; IGFBP-1 sense, CTGCGTGCAGGAGTCTGA and antisense, CCC AAAGGATGGAATGATCC.

ELISA. IGFBP-1 protein expression in the supernatant of decidualized ESCs was measured with IGFBP-1 ELISA kits (OKDA00085; Alpha Diagnostics, San Antonio, TX, USA), according to the manufacturer's protocol. The optical density, measured using a microplate reader, was put into the software to calculate the protein concentration, from which a curve was plotted using GraphPad Prism 6 software (Premier Biosoft International, Inc., Palo Alto, CA, USA).

Immunofluorescence. Fixed pESCs were incubated with anti-vimentin (1:200, ab92547) and anti-CK7 (1:200, ab181598) (both from Abcam, Cambridge, MA, USA), and 

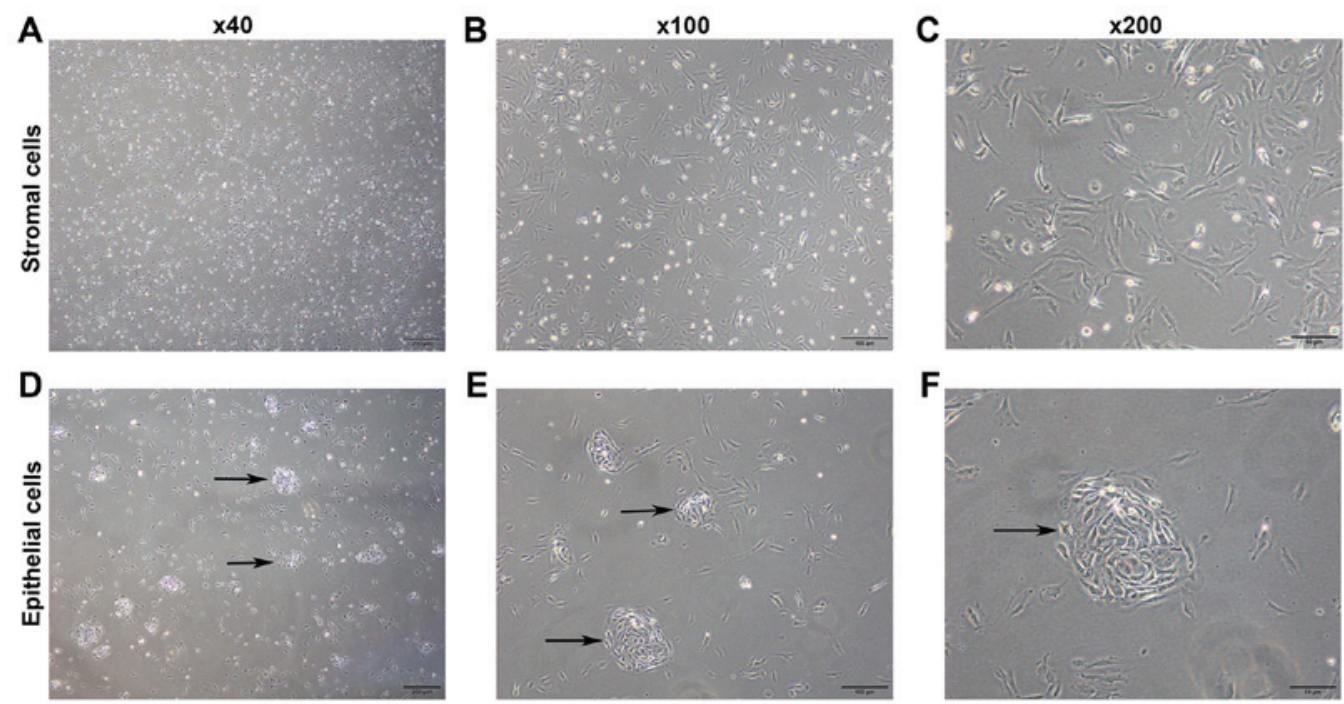

Figure 1. Primary endometrial stromal cells and glandular epithelium. Representative endometrial stromal cells at (A) x40, (B) x100 and (C) x200 magnification, and D, E and F are representative glandular epithelium at (D) x40, (E) x100 and (F) x200 magnification. Black arrows indicate glandular epithelium cells.
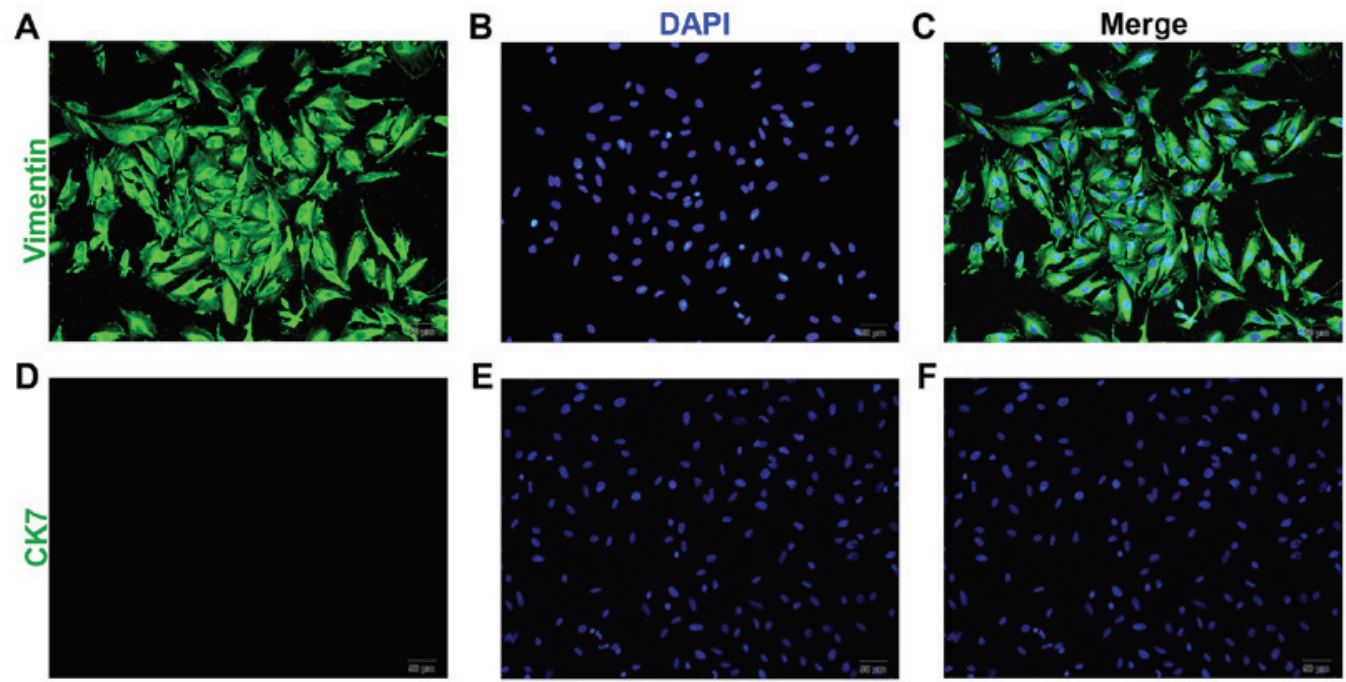

Figure 2. Primary endometrial stromal cell identification. Isolated stromal cells were stained with (A-C) stromal cell marker vimentin and (D-F) epithelium cell marker CK7 to demonstrate its purity. Magnification, x200. CK7, cytokeratin-7.

fixed ESCs from different treatments were incubated with anti-PRL (1:150, ab64377; Abcam) primary antibody overnight at $4^{\circ} \mathrm{C}$ separately, washed with PBS and incubated with FITC-conjugated secondary antibody (1:200, ab6717; Abcam). After washing with PBS, cells were mounted with anti-fading mounting medium with DAPI and observed with a fluorescence microscope (magnification, x40). Images were captured with a charge coupled device camera.

Statistical analysis. SPSS for Windows 17.0 software (SPSS Inc., Chicago, IL, USA) was used for statistical analysis. Quantitative data are presented as the mean \pm standard deviation. One-way analysis of variance was used for the analysis of single factors amongst multiple groups. The Student-Newman-Keuls and Fisher's least significant difference t-tests were used for pair-wise comparisons. $\mathrm{P}<0.05$ was considered to indicate a statistically significant difference.

\section{Results}

Morphology and identification of primary ESCs. Images of pESCs are presented in Fig. 1. Stromal cells began to adhere within several min following culture, and the adhesion was generally completed at 1-2 h of culture. Cells were triangular at an early stage following adhesion (Fig. 1A-C). The glandular epithelial cells predominantly aggregated and began to adhere at $2 \mathrm{~h}$ of culture. Furthermore, the cells exhibited a tadpole-like appearance at an early stage following adhesion, and subsequently grew into a nest shape (Fig. 1D-F).

Following 4-6 days of culture, the pESCs reached confluence and became spindle-shaped (Fig. 2A-C). Among the stromal cells, $<5 \%$ were identified as glandular epithelial cells. Following passage of the cells three times, relatively pure stromal cells were obtained, with a purity of $95 \%$, and positive vimentin (Fig. 2A-C) and negtive CK7 expression (Fig. 2D-F). 

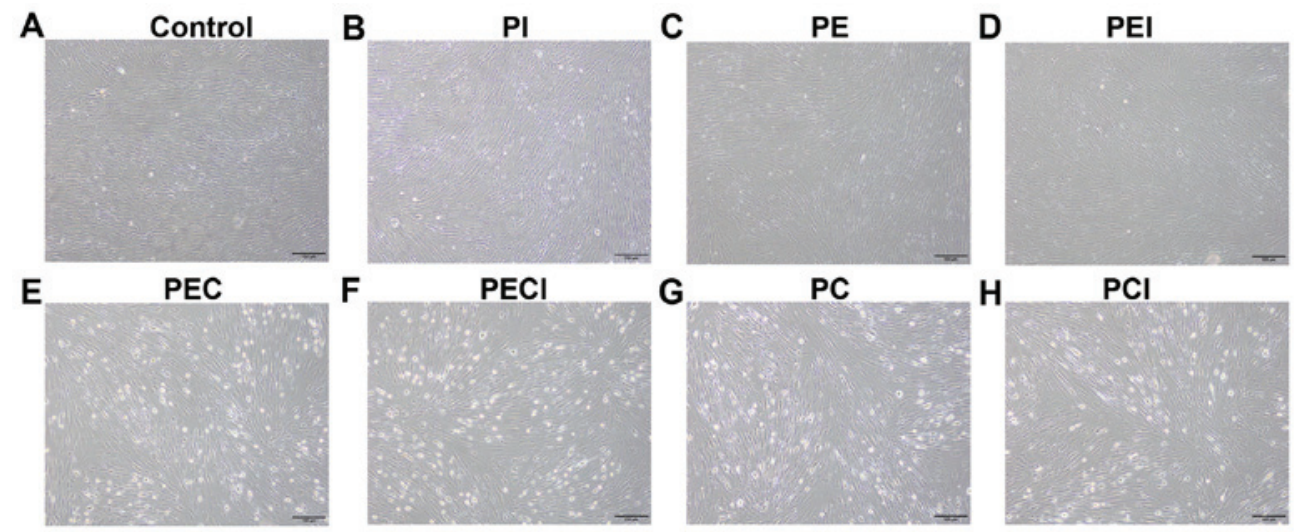

Figure 3. Morphology of decidualized stromal cells. Morphology of the stromal cells was observed under the following treatments: (A) Control, (B) PI, (C) PE, (D) PEI, (E) PEC, (F) PECI, (G) PC and (H) PCI. Magnification, x100. P4, progesterone; E2, estradiol; PI, P4+Icaritin; PE, P4+E2; PEI, P4+E2+Icaritin; PEC, P4+E2+cAMP; PECI, P4+E2+cAMP+Icaritin; PC, P4+cAMP; PCI, P4+cAMP+Icaritin; cAMP, cyclic adenosine monophosphate.

Table I. PRL mRNA expression following Icaritin treatment in decidualized cells ( $\mathrm{n}=10$ per group).

\begin{tabular}{lc}
\hline Group & Relative expression \\
\hline Control & $8.722 \times 10^{-6} \pm 1.08866 \times 10^{-5}$ \\
PI & $3.96 \times 10^{-6} \pm 2.21 \times 10^{-6}$ \\
PE & $5.82 \times 10^{-6} \pm 2.10297 \times 10^{-6}$ \\
PEI & $4.86 \times 10^{-6} \pm 1.31 \times 10^{-6}$ \\
PEC & $0.0041 \pm 0.000446$ \\
PECI & $0.002995 \pm 0.00038$ \\
PC & $0.00348 \pm 0.000416$ \\
PCI & $0.002929 \pm 0.000704$ \\
\hline
\end{tabular}

PEC vs. PECI, $q$-value $=9.831, \mathrm{P}<0.0001 ;$ PEC vs. $\mathrm{PC}$, $q$-value $=5.512, \mathrm{P}=0.0048: \mathrm{PC}$ vs. $\mathrm{PCI}, q$-value $=4.901, \mathrm{P}=0.0470$. PRL, prolactin; PI, P4+Icaritin; PE, P4+E2; PEI, P4+E2+Icaritin; PEC, P4+E2+cAMP; PECI, P4+E2+cAMP+Icaritin; PC, P4+cAMP; PCI, P4+cAMP+Icaritin; cAMP, cyclic adenosine monophosphate; $\mathrm{E} 2$, estradiol; $\mathrm{P} 4$, progesterone.

Morphological characteristics of the ESCs following decidualization. An inverted microscope was used to observe the in vitro cultured decidualized stromal cells (Fig. 3). The results revealed that the shape of the cells was irregular. The cells were polygon-shaped and revealed epithelioid changes. Multinucleation was observed for the cell nuclei, and the cell bodies were large and round in the PEC and PC groups (Fig. 3E and G) compared with the control, PI and PE groups (Fig. 3A-C), with moderate changes observed in the PEI, PECI and PCI groups (Fig. 3D, F and H).

Icaritin inhibits the expression of PRL and IGFBP-1 $\mathrm{mRNA}$ in the decidualized cells. The results revealed that PEC and $\mathrm{PC}$ were able to induce the decidualization of ESCs, as the expression of PRL (Table I) and IGFBP-1 mRNA (Table II) was significantly higher in the two groups compared with the control group, and the expression in the PEC group was also significantly higher compared with the PC group. However, the expression levels of PRL and IGFBP-1 was significantly
Table II. IGFBP-1 mRNA expression following Icaritin treatment in decidualized cells ( $\mathrm{n}=10$ per group).

\begin{tabular}{lc}
\hline Group & Relative expression \\
\hline Control & $0.000076362 \pm 3.45985 \times 10^{-5}$ \\
PI & $9.31374 \times 10^{-6} \pm 4.64574 \times 10^{-6}$ \\
PE & $1.03501 \times 10^{-5} \pm 2.74771 \times 10^{-6}$ \\
PEI & $1.68732 \times 10^{-5} \pm 3.79461 \times 10^{-6}$ \\
PEC & $0.001787477 \pm 0.000487733$ \\
PECI & $0.001435567 \pm 0.000317134$ \\
PC & $0.001470706 \pm 0.000377389$ \\
PCI & $0.000961847 \pm 0.000164622$
\end{tabular}

PEC vs. PECI, $q$-value $=4.411, \mathrm{P}=0.00318 ;$ PEC vs. $\mathrm{PC}$, $q=$ value $=3.97, \mathrm{P}=0.00219: \mathrm{PC}$ vs. $\mathrm{PCI}, q$-value $=6.378, \mathrm{P}=0.001$. IGFBP-1, insulin-like growth factor-binding protein 1; PI, P4+Icaritin; PE, P4+E2; PEI, P4+E2+Icaritin; PEC, P4+E2+cAMP; PECI, P4+E2+cAMP+Icaritin; PC, P4+cAMP; PCI, P4+cAMP+Icaritin; cAMP, cyclic adenosine monophosphate; E2, estradiol; P4, progesterone.

decreased in the PCI group compared with the PC group and in the PECI group compared with the PEC group following addition of Icaritin.

Icaritin inhibits the expression of decidualization proteins. Cell immunofluorescence of PRL revealed that the PRL was negative in the cell groups that were not decidualized in the control, PI, PE, and PEI groups (Fig. 4A-D) but positively expressed in the decidualized cells of the PEC, PECI, PC and PCI groups (Fig. 4E-H). By evaluating the decidualized marker IGFBP-1 secreted into the culture media, the IGFBP-1 protein level was demonstrated to be significantly higher in the PEC group compared with the PC group. However, the expression of IGFBP-1 protein was significantly decreased following addition of Icaritin (Table III).

\section{Discussion}

The acceptability of the growing environment of endometrium is critical to the successful implantation of embryos, and an 

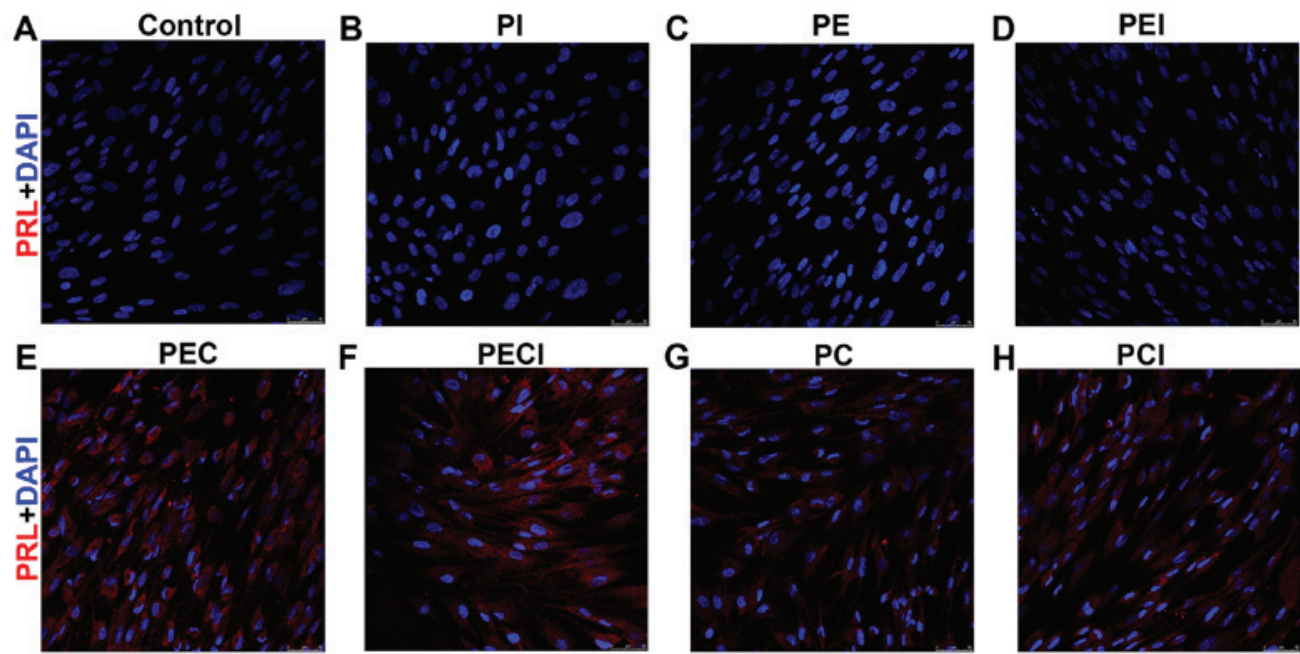

Figure 4. PRL immunofluorescent staining of the stromal cells was observed under the following treatments: (A) Control, (B) PI, (C) PE, (D) PEI, (E) PEC, (F) PECI, (G) PC and (H) PCI. Magnification, x200. PRL, prolactin; PI, P4+Icaritin; PE, P4+E2; PEI, P4+E2+Icaritin; PEC, P4+E2+cAMP; PECI, P4+E2+cAMP+Icaritin; PC, P4+cAMP; PCI, P4+cAMP+Icaritin; cAMP, cyclic adenosine monophosphate; E2, estradiol; P4, progesterone.

Table III. IGFBP-1 protein expression following Icaritin treatment in decidualized cells ( $\mathrm{n}=6$ per group).

\begin{tabular}{lr}
\hline Group & \multicolumn{1}{c}{ Relative expression } \\
\hline PEC & $10.438125 \pm 0.781819935$ \\
PECI & $9.0145 \pm 0.753969874$ \\
PC & $8.7955 \pm 1.028489461$ \\
PCI & $6.4853 \pm 0.056983423$
\end{tabular}

PEC vs. PECI, $q$-value $=5.049, \mathrm{P}=0.0023 ;$ PEC vs. $\mathrm{PC}$, $q$-value $=5.825, \mathrm{P}=0.0029: \mathrm{PC}$ vs. $\mathrm{PCI}, q$-value $=6.764, \mathrm{P}=0.0019$ IGFBP-1, insulin-like growth factor-binding protein 1; PEC, P4+E2+cAMP; PECI, P4+E2+cAMP+Icaritin; PC, P4+cAMP; PCI, P4+cAMP+Icaritin; cAMP, cyclic adenosine monophosphate; E2, estradiol; $\mathrm{P} 4$, progesterone.

endometrium that is too thin may reduce the implantation rate of embryos (3). Fatemi and Popovic-Todorovic (19) previously demonstrated that insufficient endometrium growth may result in a decreased rate of embryo implantation. The endometrium consists of ESCs and endometrial glandular epithelial cells. Usually, specimens of thin endometrium are difficult to obtain due to the low incidence of thin endometrium. In the present study, such specimens were obtained during hysterectomy for uterine fibroid or adenomyosis. High concentrations of collagenase and DNase were used in combination to digest the cells, after which the cells were filtered through a mesh and the adhesion purification method was used to separate the glandular epithelial and stromal cells. As glandular epithelial cells are difficult to passage and purify, only ESCs with relatively high purity were obtained in the present study. As the response of pESCs to hormone treatments various among pESCs derived from different patients, the T HESC cell (ESCs) line was used to investigate the effect of Icaritin on the decidualization to minimize the variation from different pESCs. Li et al (20) recently demonstrated that the use of
BerEP4-coated magnetic beads was able to assist in obtaining high-purity ESCs. Chan et al (21) previously indicated that a BerEP4 antibody exhibited specificity for luminal and glandular epithelia in the endometrium. In the present study, a $95 \%$ purity of pESCs was obtained via the adhesion culture method, which is comparable to other methods used for ESC isolation $(20,21)$.

Physiological levels of estrogen and progestogen are required for the regulation of menstruation and pregnancy maintenance (6). From the late stage of secretion, endometrial cells begin to proliferate and differentiate under the effects of estrogen and progestogen, and then undergo decidualization to form decidual cells (22). Such cells are irregular in shape; appear polygon shaped and the cell bodies become large and round, with a rich cytoplasm (6). Cell nuclei separate to demonstrate multinuclear changes (6). In humans and mice, the degree of decidualization is consistent with the degree of trophoblast invasion $(6,23,24)$. Such decidualization may then exert regulatory effects on blastocyst implantation, placenta formation and maintenance of normal pregnancy (23). If defects in decidualization exist, trophoblast invasion may be blocked and thus lead to infertility and recurrent spontaneous abortion (24). Patients with a thin endometrium exhibit a low menstrual blood volume, which maybe associated with insufficient decidualization of the endometrium (4).

Decidualization is predominantly regulated by estrogen and progestogen (6). Therefore, adding estrogen and progestogen to human or murine ESCs is able to induce a model of decidualization in vitro. Previous studies have revealed that progesterone, estrogen and cAMP co-regulate the decidualization of human ESCs in a time-dependent manner (25-27). Carden et al (28) previously used E2+P4 to treat ESCs for 14 days and successfully induce decidualization. The present study confirmed that using E2+P4 and/or cAMP to treat ESCs for $96 \mathrm{~h}$ was able to induce decidualization. Additionally, the expression of decidualization-related proteins was significantly higher in the PEC group compared with the PC group. It was speculated that this may be associated with the evidence that $\mathrm{P} 4$ serves a dominant role in the process of 
decidualization (29). However, the regulation by trace estrogen and its receptors is also required. Furthermore, the regulatory effects of estrogen are required for in vitro cultured ESCs to regulate decidualization, whereas a lower expression level of decidualization-related proteins in the PC group (compared with the PEC group) may be associated with the insufficiency of estrogen.

Previous studies have revealed that the regulatory effects of estrogen are more precise than the effects of progestogen (30-32). Icaritin has estrogenic effects, and thus maypromote the proliferation of the endometrium and improve the expression of progestogen receptors (15). Human ESCs carry progestogen and estrogen receptors, and thus can mimic the in vivo morphological and functional changes in decidualization in vitro (33). It was hypothesized that the estrogenic effects of Icaritin were able to affect the decidualization of stromal cells, and thus affect the proliferation and secretion of the endometrium. In order to avoid the individual difference in primary ESCs, E2+P4 and/or cAMP were used to treat T HESC cells in the present study in order to induce the decidualization model, after which Icaritin was added for induction. Immunostaining revealed that PRL was positively expressed, and the expression of IGFBP-1 was positively associated with the degree of decidualization. Following addition of Icaritin into the PEC and PC groups, in which decidualization was induced, the expression of PRL and IGFBP-1 mRNA and the level of IGFBP-1 protein was decreased. These observations indicated that Icaritin was able to inhibit the expression of decidualization-related genes in ESCs in vitro. Icaritin is a plant-derived estrogen that reveals anti-estrogenic effects when the estrogen level in the body is relatively high (34). Therefore, additional Icaritin in E2-induced decidualization may exert anti-estrogenic activities. Wu et al (35) recently indicated that providing excess estrogen at an early stage of pregnancy was able to inhibit the decidualization of the endometrium and lead to decidualization dysfunction. However, additional studies are required to investigate the mechanisms associated with the inhibitory effects of Icaritin on decidualization further.

\section{Acknowledgements}

The present study was supported by a grant from the Natural Science Foundation of Guangdong (grant no. 2016A030313033).

\section{References}

1. Riad ON and Hak AA: Assessment of endometrial receptivity using Doppler ultrasonography in infertile women undergoing intrauterine insemination. Gynecol Endocrinol 30: 70-73, 2014.

2. Gleicher N, Vidali A and Barad DH: Successful treatment of unresponsive thin endometrium. Fertil Steril 95: 2123.e13-e17, 2011.

3. Aydin T, Kara M and Nurettin T: Relationship between endometrial thickness and in vitro Fertilization-intracytoplasmic sperm injection outcome. Int J Fertil Steril 7: 29-34, 2013.

4. Gargett CE and Healy DL: Generating receptive endometrium in Asherman's syndrome. J Hum Reprod Sci 4: 49-52, 2011.

5. Deligdisch L: Hormonal pathology of the endometrium. Mod Pathol 13: 285-294, 2000

6. Ramathal CY, Bagchi IC, Taylor RN and Bagchi MK: Endometrial decidualization: Of mice and men. Semin Reprod Med 28: 17-26, 2010.

7. Schwab KE, Chan RW and Gargett CE: Putative stem cell activity of human endometrial epithelial and stromal cells during the menstrual cycle. Fertil Steril 84 (Suppl 2): S1124-S1130, 2005.
8. Dunn CL, Kelly RW and Critchley HO: Decidualization of the human endometrial stromal cell: An enigmatic transformation. Reprod Biomed Online 7: 151-161, 2003.

9. Kane NM, Jones M, Brosens JJ, Kelly RW, Saunders PT and Critchley HO: TGF $\beta 1$ attenuates expression of prolactin and IGFBP-1 in decidualized endometrial stromal cells by both SMAD-dependent and SMAD-independent pathways. PLoS One 5: e12970, 2010.

10. Guzel E, Buchwalder L, Basar M, Kayisli U, Ocak N, Bozkurt I, Lockwood CJ and Schatz F: Effects of tibolone and its metabolites on prolactin and insulin-like growth factor binding protein-1 expression in human endometrial stromal cells. Gynecol Endocrinol 31: 414-418, 2015.

11. Liang DK, Qi HB, Luo X, Xiao XQ and Jia XY: Comparative study of placental $\alpha$-microglobulin-1, insulin-like growth factor binding protein-1 and nitrazine test to diagnose premature rupture of membranes: A randomized controlled trial. J Obstet Gynaecol Res 40: 1555-1560, 2014.

12. Ammoun S, Schmid MC, Zhou L, Ristic N, Ercolano E, Hilton DA, Perks CM and Hanemann CO: Insulin-like growth factor-binding protein-1 (IGFBP-1) regulates human schwannoma proliferation, adhesion and survival. Oncogene 31: 1710-1722, 2012.

13. Lóránd T, Vigh E and Garai J: Hormonal action of plant derived and anthropogenic non-steroidal estrogenic compounds: Phytoestrogens and xenoestrogens. Curr Med Chem 17: 3542-3574, 2010.

14. Ma HP, Ming LG, Ge BF, Zhai YK, Song P, Xian CJ and Chen KM: Icariin is more potent than genistein in promoting osteoblast differentiation and mineralization in vitro. J Cell Biochem 112: 916-923, 2011.

15. Ye HY and Lou YJ: Estrogenic effects of two derivatives of icariin on human breast cancer MCF-7 cells. Phytomedicine 12: 735-741, 2005.

16. Pierro E, Minici F, Alesiani O, Miceli F, Proto C, Screpanti I, Mancuso S and Lanzone A: Stromal-epithelial interactions modulate estrogen responsiveness in normal human endometrium. Biol Reprod 64: 831-838, 2001.

17. Kusama K, Yoshie M, Tamura K, Kodaka Y, Hirata A, Sakurai T, Bai H, Imakawa K, Nishi H, Isaka K, et al: Regulation of decidualization in human endometrial stromal cells through exchange protein directly activated by cyclic AMP (Epac). Placenta 34: 212-221, 2013.

18. Zhang Y, Lin X, Dai Y, Hu X, Zhu H, Jiang Y and Zhang S: Endometrial stem cells repair injured endometrium and induce angiogenesis via AKT and ERK pathways. Reproduction 152: 389-402, 2016.

19. Fatemi HM and Popovic-Todorovic B: Implantation in assisted reproduction: A look at endometrial receptivity. Reprod Biomed Online 27: 530-538, 2013.

20. Li T, He H, Liu R, Wang SX and Pu DM: Isolation and identification of epithelial and stromal stem cells from eutopic endometrium of women with endometriosis. Eur J Obstet Gynecol Reprod Biol 178: 89-94, 2014.

21. Chan RW, Schwab KE and Gargett CE: Clonogenicity of human endometrial epithelial and stromal cells. Biol Reprod 70: 1738-1750, 2004

22. Franco HL, Dai D, Lee KY, Rubel CA, Roop D, Boerboom D, Jeong JW, Lydon JP, Bagchi IC, Bagchi MK and DeMayo FJ: WNT4 is a key regulator of normal postnatal uterine development and progesterone signaling during embryo implantation and decidualization in the mouse. FASEB J 25: 1176-1187, 2011.

23. Gellersen B, Brosens IA and Brosens JJ: Decidualization of the human endometrium: Mechanisms, functions, and clinical perspectives. Semin Reprod Med 25: 445-453, 2007.

24. Cha J, Sun X and Dey SK: Mechanisms of implantation: Strategies for successful pregnancy. Nat Med 18: 1754-1767, 2012.

25. Logan PC, Ponnampalam AP, Steiner M and Mitchell MD: Effect of cyclic AMP and estrogen/progesterone on the transcription of DNA methyltransferases during the decidualization of human endometrial stromal cells. Mol Hum Reprod 19: 302-312, 2012.

26. Latil A, Bièche I, Vidaud D, Lidereau R, Berthon P, Cussenot O and Vidaud M: Evaluation of androgen, estrogen (ER alpha and ER beta), and progesterone receptor expression in human prostate cancer by real-time quantitative reverse transcription-polymerase chain reaction assays. Cancer Res 61: 1919-1926, 2001.

27. Gellersen B and Brosens JJ: Cyclic decidualization of the human endometrium in reproductive health and failure. Endocr Rev 35: 851-905, 2014. 
28. Carden D, Xiao F, Moak C, Willis BH, Robinson-Jackson S and Alexander S: Neutrophil elastase promotes lung microvascular injury and proteolysis of endothelial cadherins. Am J Physiol 275: H385-H392, 1998

29. Lee K, Jeong J, Tsai MJ, Tsai S, Lydon JP and DeMayo FJ: Molecular mechanisms involved in progesterone receptor regulation of uterine function. J Steroid Biochem Mol Biol 102: 41-50, 2006.

30. Stricker R, Eberhart R, Chevailler MC, Quinn FA, Bischof P and Stricker R: Establishment of detailed reference values for luteinizing hormone, follicle, stimulating hormone, estradiol, and progesterone during different phases of the menstrual cycle on the Abbott ARCHITECT analyzer. Clin Chem Lab Med 44: 883-887, 2006

31. Tan J, Paria BC, Dey SK and Das SK: Differential uterine expression of estrogen and progesterone receptors correlates with uterine preparation for implantation and decidualization in the mouse. Endocrinology 140: 5310-5321, 1999.
32. Curtis Hewitt S, Goulding EH, Eddy EM and Korach KS: Studies using the estrogen receptor alpha knockout uterus demonstrate that implantation but not decidualization-associated signaling is estrogen dependent. Biol Reprod 67: 1268-1277, 2002.

33. Hatayama H, Kanzaki H, Iwai M, Kariya M, Fujimoto M, Higuchi T, Kojima K, Nakayama H, Mori T and Fujita J: Progesterone enhances macrophage colony-stimulating factor production in human endometrial stromal cells in vitro. Endocrinology 135: 1921-1927, 1994.

34. Kang HK, Lee SB, Kwon H, Sung CK, Park YI and Dong MS: Peripubertal administration of icariin and icaritin advances pubertal development in female rats. Biomol Ther (Seoul) 20: 189-195, 2012.

35. Bin Wu: Preliminary study on the effect of estrogen on the spontaneous pregnancy and the process of pregnancy (unpublished $\mathrm{PhD}$ thesis). Peking Union Medical College, 2014. 